\title{
A Dataset from Echocardiographic Assessment of Cardiac Function in Mice after Myocardial Infarction
}

\author{
Iordanis Mourouzis, Polixeni Mantzouratou, Constantinos Pantos ( $₫)$
}

Department of Pharmacology, University of Athens, 75 Mikras Asias Ave.,11527 Goudi, Athens, Greece

http://www.uoa.gr

\author{
ARTICLE INFO: \\ RECEIVED: 28 Jul 2015 \\ REVISED: 10 Sep 2015 \\ ACCEPTED: 30 Sep 2015 \\ ONLINE: 10 Oct 2015
}

\section{KEYWORDS:}

myocardial infarction

heart failure

cardiac remodelling

echocardiography

\begin{abstract}
Open-access datasets for cardiac remodelling after myocardial infarction in any species have not been reported, and this limits the development of systems approaches in medicine. In this paper, we present a dataset from echocardiographic assessment of male C57BL mice that were subjected to acute myocardial infarction. We present measurements that show the deterioration of global left ventricular (LV) function, regional function, development of LV dilation and hypertrophy. At the same time, we present measurements showing the degree of cardiac injury as well as the development of pulmonary congestion. Importantly, LV dilation-a hallmark of cardiac remodelling-does not develop in all subjects, and this is only partly explained by the degree of cardiac injury.
\end{abstract}

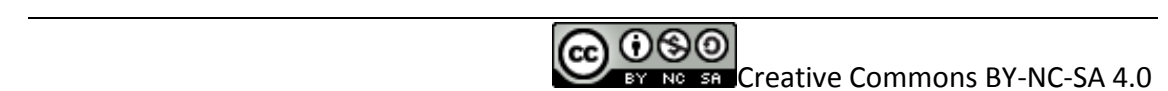

(C) 2015 Hosting by Procon Ltd. All rights reserved.

\section{Overview}

Despite the advances in current treatments, a substantial proportion of patients fail to recover after acute myocardial infarction resulting in heart failure. ${ }^{1}$ The main cause of this failure to recover is the development of cardiac remodelling, a process that leads to a series of structural and functional changes in the viable myocardium. Although progress has been made in understanding the pathophysiology of cardiac remodelling, we are still unable to predict disease progress and lack effective treatments tailored to the needs of the specific patient. ${ }^{2}$ This is due to the complex pathophysiology of the cardiac remodelling that requires a systems medicine approach through the integration of multiple data types, as well as to the complexi- ties inferring information from such data. ${ }^{3}$ To date, open-access datasets for cardiac remodelling have not been reported in any species, and this limits the development of systems medicine approaches. Our group has worked for many years in this area aiming to determine novel pathophysiologic insights and therapeutic interventions using in vivo models of myocardial infarction in mice and rats. $^{4-7}$ In this report, we present an echocardiographic dataset from male C57BL mice that were subjected to acute myocardial infarction. Part of this data has been used in previously published works that investigated the role of thyroid hormone receptor alpha 1 in cardiac remodelling, as well as the role of thyroid hormone as new therapeutic approach in this process. ${ }^{7-8}$ 
Methods

\section{Animals}

Male C57BL mice were maintained on a $12 \mathrm{~h}$ light/ dark cycle and fed with a standard chow ad libitum. Mice were handled in accordance with the Guide for the Care and Use of Laboratory Animals published by the US National Institutes of Health Guide (NIH Pub. No. 83-23, Revised 1996).

\section{Experimental model of myocardial infarction in mice}

Myocardial infarction was induced by ligation of the left coronary artery. Mice were anesthetized with sevoflurane inhalation in a closed chamber, intubated and ventilated via a tracheal cannula using a constant-volume rodent ventilator (Harvard Apparatus, Inspira, 100 breaths/min, $0.1 \mathrm{ml} / 10 \mathrm{~g}$ tidal volume). Anaesthesia was maintained by inhalation of sevoflurane (3-4\%). Left thoracotomy was performed at the fourth intercostal space followed by pericardiotomy. Left coronary artery was then ligated with a 8-0 silk round-bodied suture. The heart was quickly returned to the chest cavity, the chest was closed and mice were allowed to recover using assist mode ventilation. Atelectasis was prevented by using positive end-expiratory pressure at the end of the surgical procedure. Body temperature was maintained at $37^{\circ} \mathrm{C}$ by using a heating blanket (Harvard Homeothermic Blanket, 50-7061). The animals were left to recover for three weeks after myocardial infarction. The same procedure was followed for sham-operated animals, but the coronary artery was not ligated. Animals were 12-16 weeks old at the time of surgical procedure. A total of 16 animals were subjected to sham-operation, while 15 animals were subjected to coronary artery ligation (CAL).

\section{Experimental procedure}

Three weeks after the surgical procedure, mice were anaesthetized with ketamine hydrochloride and midazolame, subjected to echocardiography analysis, and the heart and lungs were removed. Left ventricle (LV) and right ventricle (RV) of the heart and lung weight (LW) were measured for each animal. Scar LV tissue was dissected out. The area of the scar tissue was measured in $\mathrm{mm}^{2}$ and the weight in $\mathrm{mg}$. The ratio of lung weight to body weight (LW/BW in $\mathrm{mg} / \mathrm{mg}$ ) was used to assess the development of pulmonary congestion after myocardial infarction.

\section{Echocardiography}

Short and long-axis images were acquired using a digital ultrasound system (Vivid 7 version Pro, GE Healthcare) with the $14.0-\mathrm{MHz}$ i13 L probe. A large number of consecutive measurements were performed and analysed by two independent operators. All measurements were averaged for at least 3 consecutive cardiac cycles. Left ventricular (LV) internal diameter at the diastolic phase, LV internal diameter at the systolic phase, posterior wall thickness at the diastolic phase and the ejection fraction (EF \%) were measured. EF \% was calculated using the Simpson equation. EF \% was used to determine the global contractile LV function. Systolic velocity of the posterior wall radial displacement (SVPW) was also measured. SVPW was measured from two-dimensional guided M-mode recordings obtained at the midventricular level. SVPW was calculated according to the following formula: $\mathrm{V}=\mathrm{ds} / \mathrm{dt}$, where ' $\mathrm{V}$ ' represents velocity, ' $s$ ' - the distance, and ' $\mathrm{t}$ ' represents time.

\section{Data Records}

The data in the accompanying spreadsheet is organised in the following columns:

A: It shows the ID of the subject.

B: It shows the intervention that the animals were subjected to. It includes two options - either sham-operation or coronary artery ligation (CAL).

C: It shows the duration of the time in weeks between the intervention and evaluation of measurements. All measurements were performed three weeks after the surgical procedure.

D: It shows body weight of animals in grams at the time of surgery.

E: It shows body weight of animals in grams at the day of sacrifice.

F: It shows the left ventricular (LV) end-diastolic diameter in $\mathrm{cm}$ that was measured by echocardiography.

G: It shows the left ventricular end-systolic diameter in $\mathrm{cm}$ that was measured by echocardiography.

$\mathrm{H}$ : It shows the left ventricular posterior wall thickness in $\mathrm{cm}$ that was measured by echocardiography. 
I: It shows the maximum distance from the basis to the apex of the left ventricle in $\mathrm{cm}$ (called long axis) that was measured by echocardiography.

G: It shows the left ventricular ejection fraction in percentages that was measured by echocardiography using the Simpson equation.

$\mathrm{K}$ : It shows the systolic velocity of the left ventricular posterior wall in $\mathrm{cm} / \mathrm{sec}$ that was measured by echocardiography.

L: It shows the left ventricular end-diastolic volume in $\mathrm{ml}$ that was measured by echocardiography using the Simpson equation.

M:It shows the left ventricular end-systolic volume in $\mathrm{ml}$ that was measured by echocardiography using the Simpson equation.

$\mathrm{N}$ : It shows the Wall tension index (WTI) defined as the ratio of (LV end-diastolic diameter)/ 2* (left ventricular posterior wall thickness). WTI was measured in order to indirectly assess myocardial wall stress.

O: It shows the heart rate in beats per min that was measured during echocardiography.

P: It shows the left ventricular weight in grams.

Q: It shows the weight of the scar tissue of the left ventricle in grams. Animals subjected to sham operation did not have a scar.

R: It shows the area of the scar tissue of the left ventricle in $\mathrm{mm}^{2}$. Animals subjected to sham operation did not have a scar.

S: It shows the wet lung weight in grams.

$\mathrm{T}$ : It shows the right ventricular (RV) weight in grams.

\section{Use and potential reuse}

The publication of well-structured data from the level of the cardiac cell to the level of the heart could form the basis for the development of a holistic approach to integrate different advances into a unified model of heart function and disease. ${ }^{3}$ Thus, open-access datasets referring to the process of cardiac remodelling after myocardial infarction could be valuable tools in understanding the adaptation of the heart to stress through the development of computational models. ${ }^{9}$ Furthermore, modelling the response of the heart after myocardial infarction could predict recovery of function or development of heart failure and serve as a useful tool both for developing new therapies and evaluating current treatments. In other fields, such as electrophysiology, computational models have now been developed for a variety of animal species, allowing representation of species-specific propensity for arrhythmia. ${ }^{10}$ However, models of the human cardiac myocyte that have been developed are constrained by limited available experimental data and have restricted applications. ${ }^{11-13}$

The dataset published in this paper was created after acute myocardial infarction was induced in mice by ligation of the left coronary artery. Within three weeks after myocardial infarction, the cardiac function was evaluated by echocardiography. In this data paper, we present measurements that show the deterioration of global LV function (ejection fraction), regional function (systolic velocity of posterior wall), development of LV dilation (LV end-diastolic diameter and volume) and hypertrophy (LV weight and posterior wall thickness). At the same time, we present measurements showing the degree of cardiac injury (as assessed by the scar weight and area) as well as the development of pulmonary congestion (wet lung weight and RV weight). It is interesting to note that as it happens in humans, the development of LV dilation (a hallmark of cardiac remodelling) does not appear in all subjects and this is only partly explained by the degree of cardiac injury.

\section{Conclusions}

In conclusion, we present a dataset from echocardiographic assessment of male C57BL mice that were subjected to acute myocardial infarction for 3 weeks. Similar datasets could be used to create an in silico disease model for heart failure after myocardial infarction and help to overcome the difficulty in extrapolating pre-clinical data to predict clinical efficacy.

\section{Data Files}

The data described in this paper is presented in an openly accessible spreadsheet: "01301_Mice_ Myocardial_Infarction.xIsx".

The file is available also in the Open Document Spreadsheet format.

\section{Acknowledgement}

The preparation of this manuscript was supported by the EU-funded project OpenScienceLink (Grant agreement 325101). 


\section{References}

${ }^{1}$ Springeling $\mathrm{T}$, Kirschbaum SW, Rossi A, Baks T, Karamermer $Y$, Schulz C, Ouhlous $M$, Duncker DJ, Moelker A, Krestin GP, Serruys PW, de Feyter P, van Geuns RJ. Late cardiac remodeling after primary percutaneous coronary intervention - fiveyear cardiac magnetic resonance imaging followup. Circ J. 2013;77(1):81-8. DOI: 10.1253/circj.CJ12-0043.

${ }^{2}$ Dargie H. Heart failure post-myocardial infarction: a review of the issues. Heart. 2005 May; 91(Suppl 2):ii3-ii6. DOI: 10.1136/hrt.2005.062018.

${ }^{3}$ Amanfu RK, Saucerman JJ. Cardiac models in drug discovery and development: a review. Crit Rev Biomed Eng. 2011;39(5):379-95. PMID: 22196160.

${ }^{4}$ Mourouzis I, Giagourta I, Galanopoulos G, Mantzouratou P, Kostakou E, Kokkinos AD, Tentolouris N, Pantos C. Thyroid hormone improves the mechanical performance of the postinfarcted diabetic myocardium: a response associated with up-regulation of Akt/mTOR and AMPK activation. Metabolism. 2013 Oct;62(10):1387-93. DOI: 10.1016/j.metabol.2013.05.008.

${ }^{5}$ Pantos C, Mourouzis I. Translating thyroid hormone effects into clinical practice: the relevance of thyroid hormone receptor $\alpha 1$ in cardiac repair. Heart Fail Rev. 2015 May;20(3):273-82. DOI: 10.1007/s10741-014-9465-4.

${ }^{6}$ Mourouzis I, Mantzouratou P, Galanopoulos G, Kostakou E, Dhalla AK, Belardinelli L, Pantos C. The beneficial effects of ranolazine on cardiac function after myocardial infarction are greater in diabetic than in nondiabetic rats. J Cardiovasc Pharmacol Ther. 2014 Sep;19(5):457-69. DOI: 10.1177/10742 48414524481.
${ }^{7}$ Mourouzis I, Kostakou E, Galanopoulos G, Mantzouratou P, Pantos C. Inhibition of thyroid hormone receptor $\alpha 1$ impairs post-ischemic cardiac performance after myocardial infarction in mice. Mol Cell Biochem. 2013 Jul;379(1-2):97-105. DOI: 10.1007/s11010-013-1631-9.

${ }^{8}$ Mourouzis I, Mantzouratou P, Galanopoulos G, Kostakou E, Roukounakis N, Kokkinos AD, Cokkinos DV, Pantos C. Dose-dependent effects of thyroid hormone on post-ischemic cardiac performance: potential involvement of Akt and ERK signalings. Mol Cell Biochem. 2012 Apr;363(1-2): 235-43. DOI: 10.1007/s11010-011-1175-9.

${ }^{9}$ Hunter PJ, Viceconti M. The VPH-Physiome Project: Standards and Tools for Multiscale Modeling in Clinical Applications. Biomedical Engineering IEEE Reviews 2009; 2:40-53. DOI: 10.1109/RBME. 2009.2036204.

${ }^{10}$ Williams GS, Smith GD, Sobie EA, Jafri MS. Models of cardiac excitation-contraction coupling in ventricular myocytes. Math Biosci. 2010 Jul;226 (1):1-15. DOI: 10.1016/j.mbs.2010.03.005.

${ }^{11}$ ten Tusscher KH, Noble D, Noble PJ, Panfilov AV. A model for human ventricular tissue. Am J Physiol Heart Circ Physiol. 2004 Apr; 286(4): H1573-89. DOI: 10.1152/ajpheart.00794.2003.

12 Iyer V, Mazhari R, Winslow RL. A computational model of the human left-ventricular epicardial myocyte. Biophys J. 2004 Sep; 87(3):1507-25. DOI: 10.1529/biophysj.104.043299.

${ }^{13}$ Grandi E, Pasqualini FS, Bers DM. A novel computational model of the human ventricular action potential and $\mathrm{Ca}$ transient. J Mol Cell Cardiol. 2010 Jan;48(1):112-21. DOI: 10.1016/j. yjmcc.2009.09.019. 